\title{
The theory of dual groups
}

\author{
by
}

A. Mekler and G. Schlitt (Abbotsford, B.C.)

\begin{abstract}
We study the $L_{\infty, \omega}$-theory of sequences of dual groups and give a complete classification of the $L_{\infty, \omega}$-elementary classes by finding simple invariants for them. We show that nonstandard models exist.
\end{abstract}

1. Introduction. In this paper we begin a study of dual groups and duality from a logical point of view. Recall that if $A$ is an Abelian group, its dual is the group $A^{*}=\operatorname{Hom}(A, \mathbb{Z})$, where $\mathbb{Z}$ denotes the group of integers. There is a canonical map $\sigma_{A}$ from a group $A$ to its double dual $A^{* *}=\left(A^{*}\right)^{*}$ taking an element $a$ to evaluation at $a$. The group $A$ is said to be reflexive if $\sigma_{A}$ is an isomorphism.

In his lecture series [7], Reid was interested in the structure of dual groups, and asked whether every dual group is reflexive. In recent years there have been several different constructions showing otherwise ([3], [5], [4]), and some progress has been made on other questions about their structure ([4], [8]). Here we continue this investigation by analyzing the theory of dual groups.

In fact, we will not concentrate literally on the theory of dual groups, but rather on the theory of structures derived naturally from a given dual group. For example:

Definition 1.1. A standard short sequence is a 4-sorted structure

$$
\mathfrak{A}=\left(A_{i}(i<3) ;\langle-,-\rangle ; \sigma ; \mathbb{Z}\right)
$$

for the language $(\langle-,-\rangle, \sigma)$, where $A_{0}$ is a dual group, $A_{i+1}=A_{i}^{*}, \sigma$ is the natural map from $A$ to $A^{* *}$, for $(a, f) \in A_{i} \times A_{i+1},\langle a, f\rangle=f(a)$ (for $i=0,1)$, and $\mathbb{Z}$ is a copy of the Abelian group of integers. The sequence $\mathfrak{A}$ will be called the short sequence associated with $A_{0}$.

1991 Mathematics Subject Classification: Primary 20K30; Secondary 03C75.

Research of both authors supported by NSERC Grant A8948. 
It is easy to check that for any dual group $A$, the map $\sigma_{A}$ is an embedding (such $A$ are said to be torsionless), so that $A$ may be seen as a subgroup of $A^{* *}$, which is itself a subgroup of $\mathbb{Z}^{A^{*}}$. It follows that $A$ is $\aleph_{1}$-free, that is, every countable subgroup is free. (In fact, the map $\sigma_{A}$ is even a pure embedding, so that $A$ is separable.) Thus $A$ is $L_{\infty, \omega}$-equivalent to a free group (see [1]). So in the language $L_{\infty, \omega}$, one can only distinguish between dual groups of differing rank. (Here and below, the rank of a group will take values in the set $\{0,1,2, \ldots, \infty\}$.) Hence we cannot, for example, distinguish between a nonreflexive and a reflexive dual group (both of infinite rank), an important structural division among dual groups. In the structure defined in Definition 1.1 one can do this, however; we will consider these structures in Section 2 and show exactly what may be said in the language $L_{\infty, \omega}$.

The important gain made by considering these extended structures in place of dual groups is that we are able to speak about how a dual group sits inside its double dual; this suggests extending the idea by considering the following structures:

Definition 1.2. A standard (long) sequence is an $\omega+1$-sorted structure

$$
\mathfrak{A}=\left(A_{n}(n \in \omega) ;\langle-,-\rangle ; \sigma_{n}(n \in \omega) ; \mathbb{Z}\right)
$$

for the language $\left(\langle-,-\rangle, \boldsymbol{\sigma}_{n}(n \in \omega)\right)$, in which $A_{0}$ is a dual group, $A_{i+1}=$ $A_{i}^{*}, \sigma_{n}$ is the natural map from $A_{n}$ to $A_{n+2}$, and for $(a, f) \in A_{i} \times A_{i+1}$, $\langle a, f\rangle=f(a)$ (for $i \in \omega$ ). We shall say that the sequence $\mathfrak{A}$ is associated with, or generated by, $A_{0}$.

Sometimes we will subscript the maps of a long sequence when it is necessary to distinguish between the maps of two different sequences.

In Section 3 we will give invariants for the $L_{\infty, \omega}$-theories of long sequences, which will describe exactly what the theory can say. But we also show that the nontrivial theories are undecidable (Section 4). Our work in Section 3 will enable us to show that there are nonstandard, even countable, models of the $L_{\infty, \omega}$-theory of long sequences, structures related to the "models" of [5].

For future reference, note that as we have already done in the definitions, we shall not explicitly include the language of Abelian groups in our structures. All universes of structures discussed should be regarded as Abelian groups. By "group" we will mean "Abelian group."

Suppose we have a pair of groups $A_{1}$ and $A_{2}$ along with a bilinear map $\langle-,-\rangle: A_{1} \times A_{2} \rightarrow \mathbb{Z}$. If $H$ is a subgroup of $A_{1}$, then $H^{\perp}$ will denote the subgroup of $A_{2}$ consisting of elements which annihilate $H$,

$$
H^{\perp}=\left\{f \in A_{2} \mid\langle a, f\rangle=0 \forall a \in H\right\} .
$$

(When there is a third group $A_{3}$ also acting on $A_{1}$ the location of $H^{\perp}$ will be made clear from context.) 
The book [4] contains most of what is known about dual groups. Any concepts we leave undefined here may be found there.

2. Short sequences of dual groups. In this section we show what may be said in $L_{\infty, \omega}$ about short sequences of dual groups. The methods used here will be important when we consider long sequences in Section 3. Our main result is the following

Theorem 2.1. Suppose that $A_{0}$ and $B_{0}$ are dual groups. Then $\operatorname{rank}\left(A_{0}\right)$ $=\operatorname{rank}\left(B_{0}\right)$ and $\operatorname{rank}\left(A_{2} / \sigma_{0}\left[A_{0}\right]\right)=\operatorname{rank}\left(B_{2} / \sigma_{0}\left[B_{0}\right]\right)$ iff the associated short sequences $\mathfrak{A}$ and $\mathfrak{B}$ are $L_{\infty, \omega}$-equivalent.

Pr o of. Towards a proof of "necessity", but also with an axiomatization of the theory of short sequences in mind, we isolate some facts which are true of any short standard sequence $\mathfrak{A}$. The reader may easily verify (see [1] if necessary) that all of the following statements may be formulated in $L_{\infty, \omega}$.

Axiom (i). The groups $A_{i}$ are $\aleph_{1}$-free, for $i=0,1,2$. (In particular, this implies that if $a \in A_{i}$, there is an element $a^{\prime} \in A_{i}$ so that $a=n a^{\prime}$ for some integer $n$ and $\left\langle a^{\prime}\right\rangle$ is a pure subgroup.)

Axiom (ii). If $\langle a\rangle$ is pure and nonzero in $A_{i}$, then there exists $f \in A_{i+1}$ so that $\langle a, f\rangle=1$ (for $i=0,1$ ).

Axiom (iii). If $\langle f\rangle$ is pure and nonzero in $A_{i+1}$ then there is $a \in A_{i}$ so that $\langle a, f\rangle=1$ (for $i=0,1)$.

Axiom (iv). $\langle a-b, f\rangle=\langle a, f\rangle-\langle b, f\rangle$ for all $a, b \in A_{i}$ and $f \in A_{i+1}$.

Axiom (v). $\langle a, f-g\rangle=\langle a, f\rangle-\langle a, g\rangle$ for all $a \in A_{i}$ and $f, g \in A_{i+1}$.

Axiom (vi). $\langle a, f\rangle=\langle f, \sigma(a)\rangle$. (Notice that Axioms (ii)-(v) imply that $\sigma$ is a pure embedding.)

Axiom (vii). If $H$ is a finite rank pure subgroup of $A_{2} / \sigma_{0}\left[A_{0}\right]$ then $\left(A_{2} / \sigma_{0}\left[A_{0}\right]\right) / H$ is $\aleph_{1}$-free.

(Axiom (vii) holds for any standard sequence since $\sigma_{0}\left[A_{0}\right]$ splits $A_{2}$, by Lemma 3.3.)

Notice that the proof of "necessity" is trivial if $\operatorname{rank}\left(A_{0}\right)$ is finite, for then $\mathfrak{A}$ and $\mathfrak{B}$ are isomorphic. Suppose then that $\operatorname{rank}\left(A_{0}\right)=\infty$.

We define a set $P$ of isomorphisms between substructures of $\mathfrak{A}$ and $\mathfrak{B}$ and show that it has the back-and-forth property. This suffices to show that $\mathfrak{A}$ and $\mathfrak{B}$ are $L_{\infty, \omega}$-equivalent (see [1]).

The set $P$ consists of isomorphisms $\phi=\left(\phi_{0}, \phi_{1}, \phi_{2}\right)$ between substructures $\mathfrak{F}=\left(F_{0}, F_{1}, F_{2}\right)$ of $\mathfrak{A}$ and $\mathfrak{G}=\left(G_{0}, G_{1}, G_{2}\right)$ of $\mathfrak{B}$ with the following properties: 
(A) The groups $F_{i}$ are finite-rank pure subgroups of $A_{i}$ (for $i=0,1,2$ ). (And likewise for the $G_{i}$.)

(B) The groups $F_{0}$ and $F_{1}$ have dual bases, that is, bases $\left\{a_{1}, \ldots, a_{n}\right\}$ and $\left\{f_{1}, \ldots, f_{n}\right\}$ so that $\left\langle a_{i}, f_{j}\right\rangle=0$ if $i \neq j$ and 1 otherwise. (And likewise for the $G_{i}$.)

(C) $\quad \sigma\left[F_{0}\right]=F_{2} \cap \sigma\left[A_{0}\right]$, and likewise for $\mathfrak{G}$.

(D) $\quad\left(F_{2}+\sigma\left[A_{0}\right]\right) / \sigma\left[A_{0}\right]$ is pure in $A_{2} / \sigma\left[A_{0}\right]$ and of the same rank as $G_{2}+\sigma\left[B_{0}\right] / \sigma\left[B_{0}\right]$ (which is pure in $B_{2} / \sigma\left[B_{0}\right]$ ).

Note that (B) implies that $F_{0} \oplus F_{1}^{\perp}=A_{0}$ and $F_{1} \oplus F_{0}^{\perp}=A_{1}$ (and likewise for $\mathfrak{B})$.

Assume then that we have an isomorphism $\phi$ satisfying statements (A) through (D). We must show how to extend $\phi$ when an element is added to $F_{0}, F_{1}$ or $F_{2}$. (The cases for the $G_{i}$ are symmetric.) The reader should be careful to note that our arguments use only properties implied by Axioms (i) through (vii).

Case I: We pick $a \in A_{0}-F_{0}$.

Write $a=a_{0}+a_{1}$ where $a_{0} \in F_{0}$ and $a_{1} \in F_{1}^{\perp}$, and $a_{1}=n a_{2}$ for some integer $n$, so that $\left\langle a_{2}\right\rangle$ is pure. Since $\sigma\left[B_{0}\right] /\left(\sigma\left[B_{0}\right] \cap G_{2}\right)$ is of infinite rank (by hypothesis), we may choose $b_{2} \in G_{1}^{\perp}$ so that $\left\langle b_{2}\right\rangle$ is pure and $\sigma\left(b_{2}\right) \notin G_{2}$. Now that we have $b_{2}$, pick $f \in F_{0}^{\perp}$ so that $\left\langle f, a_{2}\right\rangle=1$ and $g \in G_{0}^{\perp}$ so that $\left\langle g, b_{2}\right\rangle=1$ (using Axiom (ii)). Now extend $F_{0}$ to $F_{0} \oplus\left\langle a_{2}\right\rangle, F_{1}$ to $F_{1} \oplus\langle f\rangle$ and $F_{2}$ to $F_{2}+\left\langle\sigma\left(a_{2}\right)\right\rangle$, and likewise for the $G_{i}$. Extend $\phi$ to $\phi^{\prime}$ by letting $\phi^{\prime}$ take $a_{2}$ to $b_{2}, f$ to $g$, and $\sigma\left(a_{2}\right)$ to $\sigma\left(b_{2}\right)$. It is clear that hypotheses (B), (C) and (D) are still met in the extended structures. Obviously requirement (A) still holds for $i=1,2$; we check that $F_{2}+\left\langle\sigma\left(a_{2}\right)\right\rangle$ is pure in $A_{2}$. (The case for $G_{2}$ is similar.)

Suppose then that $h \in A_{2}$ and $n h=j+m \sigma\left(a_{2}\right)$ for some $j \in F_{2}$ and $m \in \mathbb{Z}$. By hypothesis (D) on $\mathfrak{F}$, we know that $h \in F_{2}+\sigma\left[A_{0}\right]$, so that $h=k+\sigma(c)$ for some $k \in F_{2}$ and $c \in A_{0}$. Then

$$
n k-j+n \sigma(c)-m \sigma\left(a_{2}\right)=0
$$

so that $n \sigma(c)-m \sigma\left(a_{2}\right)$ is in $F_{2}$, and therefore by hypothesis $(\mathrm{C})$ on $\mathfrak{F}$, is in $\sigma\left(F_{0}\right)$. Thus $n \sigma(c) \in \sigma\left[F_{0}+\left\langle a_{2}\right\rangle\right]$. Now $\sigma\left[F_{0}+\left\langle a_{2}\right\rangle\right]$ is a pure subgroup of $A_{2}$ by hypothesis (A) on $\mathfrak{F}$, the choice of $a_{2}$, and the fact that $\sigma$ is a pure embedding (Axiom (vi)). Thus $\sigma(c) \in \sigma\left[F_{0}+\left\langle a_{2}\right\rangle\right]$, so that $h \in F_{2}+\left\langle\sigma\left(a_{2}\right)\right\rangle$.

Since $a$ is in the domain of $\phi^{\prime}$, we are done. This concludes Case I.

For the next case we will need the following lemma, due to Chase [2] (or see Theorem XI.3.2 in [4]).

Lemma 2.2. Suppose that $A_{1}$ and $A_{2}$ are groups, and $\langle-,-\rangle: A_{1} \times A_{2} \rightarrow$ $\mathbb{Z}$ is a bilinear map so that Axioms (i)-(v) are satisfied. If $a_{1}, \ldots, a_{n}$ are independent elements of $A_{1}$ which generate a pure subgroup, and $m_{1}, \ldots, m_{n}$ 
are integers, then there is an element $f \in A_{2}$ so that $\left\langle f, a_{i}\right\rangle=m_{i}$ for $i=1, \ldots, n$.

Case II: We pick $f \in A_{1}-F_{1}$.

Write $f=f_{0}+f_{1}$ where $f_{0} \in F_{1}, f_{1} \in F_{0}^{\perp}$, and $f_{1}=n f_{2}$ so that $\left\langle f_{2}\right\rangle$ is pure. Next, pick $a \in F_{1}^{\perp}$ so that $\left\langle a, f_{2}\right\rangle=1$ (so that $\langle a\rangle$ is pure). Now we may engage Case I, finding a $b \in G_{1}^{\perp}$ corresponding to $a$. Extend the map $\phi_{2}$ on $F_{2}$ to an isomorphism $\phi_{2}^{\prime}$ from $F_{2}+\langle\sigma(a)\rangle$ to $G_{2}+\langle\sigma(b)\rangle$ in the obvious way (possible since $\sigma(a)$ is independent of $F_{2}$, by hypothesis $(\mathrm{C})$ ). Now we may apply Lemma 2.2 to find a $g_{2} \in B_{1}$ so that $\left\langle g_{2}, \phi_{2}^{\prime}(h)\right\rangle=\left\langle f_{2}, h\right\rangle$ for all $h \in F_{2}+\langle\sigma(a)\rangle$. Then $g_{2} \in G_{0}^{\perp}$ and $\left\langle b, g_{2}\right\rangle=1$, so that the extension $G_{1} \oplus\left\langle g_{2}\right\rangle$ is pure in $B_{2}$. We extend the other groups in the obvious way, and complete the extension of $\phi$ to an isomorphism between the extended structures, just as in Case I. As above, one can check that requirements (A) to (D) are still satisfied.

Case III: We pick $k \in A_{2}-F_{2}$.

There are two possibilities:

C a s e III(a): If $k \in F_{2}+\sigma_{0}\left[A_{0}\right]$, we may add the appropriate element to $A_{0}$, proceeding as in Case I.

Case III(b): $k \notin F_{2}+\sigma_{0}\left[A_{0}\right]$. Notice that

$$
\frac{A_{2} / \sigma\left[A_{0}\right]}{\left(F_{2}+\sigma\left[A_{0}\right]\right) / \sigma\left[A_{0}\right]}
$$

is $\aleph_{1}$-free, by induction hypothesis (D) and Axiom (vii). So we may choose $k_{1}$ in $A_{2}$ so that $k+F_{2}+\sigma\left[A_{0}\right]=n\left(k_{1}+F_{2}+\sigma\left[A_{0}\right]\right)$ for some integer $n$, and $\left\langle k_{1}+F_{2}+\sigma\left[A_{0}\right]\right\rangle$ is pure in $A_{2} /\left(F_{2}+\sigma\left[A_{0}\right]\right)$. By hypothesis (D), and theorem hypothesis, we may choose $l_{1} \in B_{2}$ so that $\left\langle l_{1}+G_{2}+\sigma\left[B_{0}\right]\right\rangle$ is pure and nonzero in $B_{2} /\left(G_{2}+\sigma\left[B_{0}\right]\right)$. Applying Lemma 2.2 , we may choose $b \in G_{0}$ so that $\left\langle\phi_{1}(f), \sigma(b)+l_{1}\right\rangle=\left\langle f, k_{1}\right\rangle$, for all $f \in F_{1}$. Finally, we extend $G_{2}$ to $G_{2}+\left\langle\sigma(b)+l_{1}\right\rangle, F_{2}$ to $F_{2}+\left\langle k_{1}\right\rangle$, and $\phi$ to an isomorphism between the extended structures taking $k_{1}$ to $\sigma(b)+l_{1}$. Our choice of $k_{1}$ and $l_{1}$ ensure that requirement $(\mathrm{D})$ is still met, and the other requirements obviously still hold.

Now we may engage Case III(a) to make the necessary extension to an isomorphism with $k$ in its domain. This completes Case III and the proof of the "necessity" direction.

It is easy to see that two short sequences $\mathfrak{A}$ and $\mathfrak{B}$ which are $L_{\infty, \omega^{-}}$ equivalent must satisfy the rank conditions in the hypothesis.

Corollary 2.3. Axioms (i)-(vii) axiomatize the $L_{\infty, \omega}$-theory of short sequences of dual groups. 
Proof. Suppose $\mathfrak{B}$ is some structure $\left(B_{i}(i<3) ;\langle-,-\rangle ; \sigma ; \mathbb{Z}\right)$ which satisfies Axioms (i)-(vii). Let $\mathfrak{A}$ be a short standard sequence for which $\operatorname{rank}\left(A_{0}\right)=\operatorname{rank}\left(B_{0}\right)$ and $\operatorname{rank}\left(A_{2} / \sigma\left[A_{0}\right]\right)=\operatorname{rank}\left(B_{2} / \sigma\left[B_{0}\right]\right)$. (There are many constructions of such groups $A_{0}$, see for example [3], [4], [8].) Then we may carry out the argument in the proof of Theorem 2.1 to see that $\mathfrak{B}$ and $\mathfrak{A}$ are $L_{\infty, \omega}$-equivalent. It follows that $\mathfrak{B}$ models the $L_{\infty, \omega}$-theory of short sequences of dual groups.

Since a dual group $A_{0}$ is a direct summand (under $\sigma$ ) of its double dual $A_{2}$ (Lemma 3.3), it may seem convenient to include in the structure of a short sequence a splitting $\varrho: A_{2} \rightarrow A_{0}$, so that $\varrho \sigma=\operatorname{Id}_{A_{0}}$. Then in the proof of Theorem 2.1, Case III would seem easier to establish. Doing the analogous thing for long sequences will be especially tempting in Section 3. But for this strengthened notion of a short sequence, Theorem 2.1 does not hold:

EXAmple 1. Denote by $G$ the torsionless but not separable group $\mathbb{Z}^{(\omega)}+$ $2 \mathbb{Z}^{\omega}$, a subgroup of $\mathbb{Z}^{\omega}$. It is not hard to see that $G^{*}=\mathbb{Z}^{(\omega)}$. Let $A$ be a torsionless group such that $A^{* *} / \sigma[A] \cong G([8])$ and let $B$ be a torsionless group such that $B^{* *} / \sigma[B] \cong \mathbb{Z}^{(\omega)}$. Now consider the augmented short sequences

$$
\mathfrak{A}=\left(A_{i}(i<3) ; \sigma ; \varrho_{\mathfrak{A}} ; \mathbb{Z}\right) \text { and } \mathfrak{B}=\left(B_{i}(i<3) ; \sigma ; \varrho_{\mathfrak{B}} ; \mathbb{Z}\right)
$$

in which $A_{0}=A^{*}, B_{0}=B^{*}$, and $\varrho_{\mathfrak{A}}$ and $\varrho_{\mathfrak{B}}$ are the corresponding restriction maps. (That is, for $a \in A$ and $f \in A_{2}, \varrho_{\mathfrak{A}}(f)(a)=f\left(\sigma_{A}(a)\right)$, and likewise for $B$.)

Then we have $\operatorname{rank}\left(A_{0}\right)=\operatorname{rank}\left(B_{0}\right)$, and since $A_{2} / \sigma\left[A_{0}\right] \cong G^{*}$ (Lemma 3.3) (and similarly for $\mathfrak{B}), \operatorname{rank}\left(A_{2} / \sigma\left[A_{0}\right]\right)=\operatorname{rank}\left(B_{2} / \sigma\left[B_{0}\right]\right)$. But $\mathfrak{A}$ and $\mathfrak{B}$ are not $L_{\infty, \omega}$-equivalent. For the fact that $G$ and $\mathbb{Z}^{(\omega)}$ are torsionless ensures that the subgroup of $A_{1}\left(B_{1}\right)$ annihilated by the kernel of $\varrho_{\mathfrak{A}}\left(\varrho_{\mathfrak{B}}\right)$ is $\sigma_{A}[A]\left(\sigma_{B}[B]\right)$. Thus we can say in $L_{\infty, \omega}$ sentences about $\mathfrak{A}$ and $\mathfrak{B}$ that $A_{1} / \operatorname{ker}\left(\varrho_{\mathfrak{A}}\right)^{\perp}(\cong G)$ is not separable, while $B_{1} / \operatorname{ker}\left(\varrho_{\mathfrak{B}}\right)^{\perp}\left(\cong \mathbb{Z}^{(\omega)}\right)$ is separable. So $\mathfrak{A}$ and $\mathfrak{B}$ are not $L_{\infty, \omega}$-equivalent.

We will return to these observations in Section 4.

3. Sequences of dual groups. Here we consider the $L_{\infty, \omega}$-theories of the long sequences defined in Definition 1.2, and prove a result analogous to Theorem 2.1. We will need the following definition from [4].

Definition 3.1. Given a dual group $A$, by induction on $n$ define $D^{0}(A)$ $=A$, and $D^{n+1}(A)=\left(D^{n}(A)\right)^{* *} / \sigma\left[D^{n}(A)\right]$. Then the length rank of $A$ is the pair $(n, m)$ of elements of $\omega \cup\{\infty\}$ where $n=\sup \left\{k \mid D^{k}(A) \neq 0\right\}$, $m=\infty$ if $n=\infty$ and $\operatorname{rank}\left(D^{n}(A)\right)$ otherwise. (We set $\sup \emptyset=0$.)

TheOREM 3.2. The length rank of $A_{0}$ is equal to the length rank of $B_{0}$ iff the associated sequences $\mathfrak{A}$ and $\mathfrak{B}$ are $L_{\infty, \omega}$-equivalent. 
Towards a proof of this we make the following definitions. Given a (long) sequence $\mathfrak{A}$, we define the quotient sequence $\mathfrak{A}_{1}$ with universe $A_{1, n}(n \in \omega)$, by $A_{1,0}=A_{2} / \sigma_{0}\left[A_{0}\right]$ and $A_{1, n}=\left(\sigma_{n-1}\left[A_{n-1}\right]\right)^{\perp}$ (a subgroup of $A_{n+2}$ ) for $n \geq 1$. We denote the projection map from $A_{2}$ to $A_{2} / \sigma_{0}\left[A_{0}\right]$ by $\pi$ and define the bracket map for $\mathfrak{A}_{1}$ as follows: Given $a \in A_{1, n}$ and $f \in A_{1, n+1}$, we let

$$
\langle a, f\rangle_{1}= \begin{cases}\langle a, f\rangle_{\mathfrak{A}} & \text { if } n \geq 1 \\ \left\langle a^{\prime}, f\right\rangle_{\mathfrak{A}}\left(\text { where } \pi\left(a^{\prime}\right)=a\right) & \text { if } n=0 .\end{cases}
$$

We claim that with this bracket map, each group $A_{1, n+1}$ may be seen as the dual of $A_{1, n}$. This follows easily from repeated applications of the following (purely categorical) result of [4]:

Lemma 3.3. Suppose that $A$ is a dual group, $A=B^{*}$. Then $A$ splits $A^{* *}$ via $\sigma_{A}$. That is, there is a homomorphism $\varrho: A^{* *} \rightarrow A$ so that $\varrho \sigma=\operatorname{Id}_{A}$. Also, $\operatorname{ker}(\varrho)=\left(\sigma_{B}[B]\right)^{\perp}$, and this kernel is itself a dual group.

Proof. Let $\varrho$ be the restriction map, that is, $\langle b, \varrho(f)\rangle=\left\langle\sigma_{B}(b), f\right\rangle$ for all $f \in A^{* *}$ and $b \in B$. Then $\varrho \sigma=\operatorname{Id}_{A}$, clearly $\operatorname{ker}(\varrho)=\left(\sigma_{B}[B]\right)^{\perp}$, and it is straightforward to show that $\left(\sigma_{B}[B]\right)^{\perp} \cong\left(A^{*} / \sigma_{B}[B]\right)^{*}$.

Applying the lemma also establishes that $A_{1,0}$ is a dual group. Now since each group is the dual of the previous, we may define the $\sigma$ maps appropriately, to obtain a standard sequence,

$$
\mathfrak{A}^{\prime}=\left(A_{1, n}(n \geq 0) ;\langle-,-\rangle_{1} ; \sigma_{1, n}(n \geq 0) ; \mathbb{Z}\right),
$$

isomorphic to the sequence associated with $A_{2} / \sigma_{0}\left[A_{0}\right]$.

We may extend the definition above, and define by induction the $n$th quotient model $\mathfrak{A}_{n}$ for $n \geq 0$ setting $\mathfrak{A}_{0}$ equal to $\mathfrak{A}$ and $\mathfrak{A}_{n+1}$ equal to the quotient sequence of $\mathfrak{A}_{n}$. For $n \geq 0$ we will denote the $m$ th group in $\mathfrak{A}_{n}$ by $A_{n, m}$, the $m$ th $\sigma$ map by $\sigma_{n, m}$, and the bracket map by $\langle-,-\rangle_{n}$ (or possibly just $\langle-,-\rangle$ when no confusion is possible).

Let $\pi_{n}$ be the projection map linking $\mathfrak{A}_{n}$ with $\mathfrak{A}_{n+1}$.

With quotient models in hand, we further define the derived sequence of a sequence $\mathfrak{A}$. This is an $\omega+1$-sorted structure

$$
\mathcal{A}=\left(A_{n, i}(n \in \omega, i<3) ;\langle-,-\rangle, \sigma_{n}, \pi_{n}, \mathbb{Z}\right)
$$

for the language $\left(\langle-,-\rangle ; \boldsymbol{\sigma}_{n}(n \geq 0) ; \boldsymbol{\pi}_{n}(n \geq 0)\right)$ for which

(i) $\sigma_{n}: A_{n, 0} \rightarrow A_{n, 2}$ has the same action as $\sigma_{n, 0}$ in the $n$th quotient model $\mathfrak{A}_{n}$.

(ii) $\langle-,-\rangle: A_{n, i} \times A_{n, i+1} \rightarrow \mathbb{Z}(i=0,1)$ has the same action as $\langle-,-\rangle_{n}$ restricted to $A_{n, i} \times A_{n, i+1}$ in $\mathfrak{A}_{n}$. 
(iii) $\pi_{n}: A_{n, 2} \rightarrow A_{n+1,0}$ has the action described above.

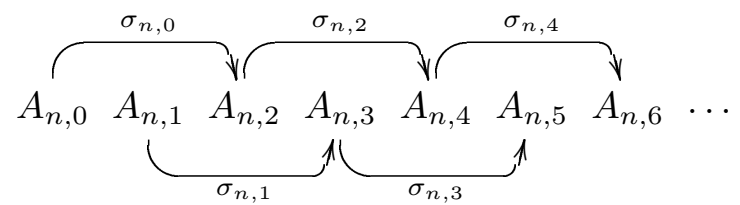

The $n$th quotient model $\mathfrak{A}_{n}$ (bracket maps not shown)

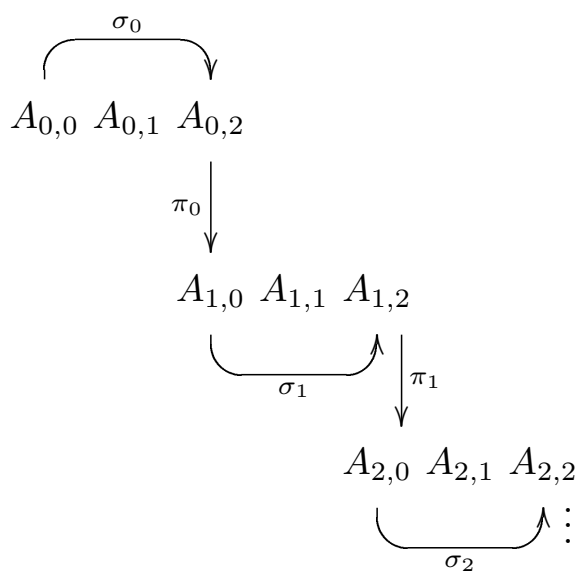

In the sequel, we will reserve Fraktur letters for structures with the type of long sequences, and calligraphic letters for structures with the type of derived sequences. By the length rank of a structure

$$
\mathcal{A}=\left(A_{n, i}(n \in \omega, i<3) ;\langle-,-\rangle, \sigma_{n}, \pi_{n}, \mathbb{Z}\right)
$$

we mean the pair $(n, m)$ where $n$ is the greatest integer such that $A_{n, 0} \neq\{0\}$ (or $\infty$ if no such integer exists), and $m=\infty$ if $n=\infty$ or $\operatorname{rank}\left(A_{n, 0}\right)$ otherwise. Thus the derived sequence $\mathcal{A}$ of the sequence $\mathfrak{A}$ generated by a group $A_{0}$ of length rank $(n, m)$ is of length rank $(n, m)$.

Lemma 3.4. If the derived sequences $\mathcal{A}$ and $\mathcal{B}$ of two models $\mathfrak{A}$ and $\mathfrak{B}$ are isomorphic, then $\mathfrak{A}$ and $\mathfrak{B}$ are isomorphic.

P r o o f. We show how to recover a model $\mathfrak{A}$ from its derived sequence $\mathcal{A}$. For later purposes we will isolate a portion of this proof, and set it in a more general context:

Construction Technique. We present a technique for transforming a structure of the type of a derived sequence,

$$
\mathcal{A}=\left(A_{n, i}(n \in \omega, i<3) ;\langle-,-\rangle_{\mathcal{A}}, \sigma_{\mathcal{A}, n}, \pi_{n}, \mathbb{Z}\right),
$$

into a structure with the type of a long sequence,

$$
\mathfrak{A}=\left(A_{n}(n \in \omega) ;\langle-,-\rangle_{\mathfrak{A}} ; \sigma_{\mathfrak{A}, n}(n \in \omega) ; \mathbb{Z}\right) .
$$


The definition of the $A_{i}$ proceeds by induction on $i$. First, we let $A_{i}=$ $A_{0, i}(i=0,1,2)$. Now suppose $i \geq 3$, and we have given the technique for constructing the groups $A_{j}$ for $j<i$. Let $A_{i}=A_{i-2} \oplus A_{i-2}^{\prime}$ where $A_{i-2}^{\prime}$ is the result of applying the construction technique to the truncated structure

$$
\mathcal{A}^{\prime}=\left(A_{n, i}(n \geq 1, i<3) ;\langle-,-\rangle_{\mathcal{A}}, \sigma_{\mathcal{A}, n}, \pi_{n}, \mathbb{Z}\right) .
$$

Define the map $\tau_{i}: A_{i} \rightarrow A_{i-2}^{\prime}$ to be $\pi_{0}$ if $i=2$ and the natural projection if $i>2$. Thus for example in the following diagram the top row consists of the groups $A_{i}$ for $0 \leq i \leq 5$ and the bottom row consists of the groups $A_{i}^{\prime}$ for $0 \leq i \leq 3$.

$$
\begin{aligned}
& \begin{array}{ccccc}
A_{0,0} & A_{0,1} & A_{0,2} & \begin{array}{c}
A_{0,1} \\
\oplus
\end{array} & A_{0,2} \\
A_{1,1} & A_{1,2}
\end{array} \quad\left(\begin{array}{c}
A_{0,1} A_{1,1} \\
\oplus \oplus \oplus \\
A_{1,1} A_{2,1}
\end{array}\right) \\
& \tau_{2} \downarrow \tau_{3} \downarrow \quad \tau_{4} \mid \quad \tau_{5} \downarrow \\
& A_{1,0} \quad A_{1,1} \quad A_{1,2} \quad \stackrel{A_{1,1}}{\oplus}
\end{aligned}
$$

We define the bracket map as follows. For $i=0,1$ and $(a, b) \in A_{i} \times A_{i+1}$, we let $\langle a, b\rangle_{\mathfrak{A}}=\langle a, b\rangle_{\mathcal{A}}$. Suppose $i \geq 2$ and that we have shown how to define the bracket map for all $j<i$. Given $(a, b)$ as above, write $b=c+d$ where $c \in A_{i-1}$ and $d \in A_{i-1}^{\prime}$. Now let

$$
\langle a, b\rangle_{\mathfrak{A}}=\langle c, a\rangle_{\mathfrak{A}}+\left\langle\tau_{i}(a), d\right\rangle_{\mathfrak{A}_{1}}
$$

where $\mathfrak{A}_{1}$ is the model being constructed from $\mathcal{A}^{\prime}$. Finally, we let $\sigma_{\mathfrak{A}, 0}=\sigma_{\mathcal{A}, 0}$ and $\sigma_{\mathfrak{A}_{i}}$ be inclusion for $i \geq 1$. This completes the construction technique.

We now show that given a long sequence $\mathfrak{A}$, if we apply the construction procedure to the derived sequence $\mathcal{A}$, we obtain a sequence isomorphic to $\mathfrak{A}$. This will establish Lemma 3.4.

Given a standard long sequence $\mathfrak{A}$, a $k$-truncation of $\mathfrak{A}$ is the structure

$$
\left(A_{n}(n<k) ;\langle-,-\rangle ; \sigma_{n}(n<k-2) ; \mathbb{Z}\right) .
$$

We show by induction that for all $k$ the $k$-truncation of $\mathfrak{A}$ is isomorphic to the $k$-truncation of the sequence resulting from applying the construction procedure to $\mathcal{A}$. Since the isomorphisms for various $k$ are compatible, the result follows immediately.

The cases for $k=1,2$ or 3 are trivial, by definition of the construction procedure. Suppose that $l \geq 3$, and that the result holds for every integer 
$1 \leq k<l$. Now the derived sequence $\mathcal{A}^{\prime}$ of the quotient model $\mathfrak{A}_{1}$ is equal (via an obvious shift) to the structure

$$
\left(A_{n, i}(n \geq 1, i<3) ;\langle-,-\rangle, \sigma_{n}, \pi_{n}, \mathbb{Z}\right),
$$

and so applying the induction hypothesis, the construction procedure reconstructs the $(l-1)$-truncation of $\mathfrak{A}_{1}$. Now Lemma 3.3 says that $A_{l-2} \oplus A_{l-2}^{\prime}$ is isomorphic to $A_{l}$, so that the construction procedure applied to $\mathcal{A}$ does give the correct group in the $l$ th position. One may also use the lemma to easily check that the procedure gives the correct $\sigma$ and bracket maps.

We may now prove Theorem 3.2.

Proof (necessity). Suppose that the dual groups $A_{0}$ and $B_{0}$ have the same length rank. Form the two corresponding standard models $\mathfrak{A}$ and $\mathfrak{B}$. To show that these two models are $L_{\infty, \omega}$-equivalent we will first show that their derived sequences $\mathcal{A}$ and $\mathcal{B}$ are $L_{\infty, \omega}$-equivalent, by an argument similar to that for the short sequences in Section 1 . Then by taking a forcing extension which collapses the cardinalities of $\mathfrak{A}$ and $\mathfrak{B}$ to $\aleph_{0}$, we will know by Lemma 3.4 that they are isomorphic in the extended universe. It follows (via a standard argument, or see [6]) that they are $L_{\infty, \omega}$-equivalent in the ground model. (This forcing argument may be replaced with a back-andforth argument, but at the cost of greater complexity of proof.)

In the nontrivial case, we assume that $A_{0}$ and $B_{0}$ are of infinite rank.

It follows directly from the definition of length rank that

$$
\operatorname{rank}\left(A_{n, 2} / \sigma_{n}\left[A_{n, 0}\right]\right)=\operatorname{rank}\left(B_{n, 2} / \sigma_{n}\left[B_{n, 0}\right]\right)
$$

for all $n$. We show that we can build a set of partial isomorphisms between substructures of $\mathcal{A}$ and $\mathcal{B}$ which satisfy the back-and-forth property, primarily by appealing to the short sequence case.

As we did in the case of short sequences, we shall list various properties ("axioms") that any sequence derived from a long sequence must have. In our proof we shall use only these properties, a fact we will appeal to in the next section.

(i) Each $A_{n, i}$ is an $\aleph_{1}$-free Abelian group.

(ii) If $\langle a\rangle$ is pure and nonzero in $A_{n, i}$, then there exists $f \in A_{n, i+1}$ so that $\langle a, f\rangle=1$ (for $i=0,1)$.

(iii) If $\langle f\rangle$ is pure and nonzero in $A_{n, i+1}$, then there exists $f \in A_{n, i}$ so that $\langle a, f\rangle=1$ (for $i=0,1)$.

(iv) For $a \in A_{n, i}, f, g \in A_{n, i+1},\langle a, f-g\rangle=\langle a, f\rangle-\langle a, g\rangle$ (for $i=0,1$ ).

(v) For $a, b \in A_{n, i}, f \in A_{n, i+1},\langle a-b, f\rangle=\langle a, f\rangle-\langle b, f\rangle$.

(vi) $\langle a, f\rangle=\left\langle\sigma_{n}(a), f\right\rangle$ for all $a \in A_{n, i}, f \in A_{n, i+1}$.

(vii) $\pi_{n}$ is a surjection with kernel $\sigma_{n}\left[A_{n, 0}\right]$. 
Our set $P$ of partial isomorphisms will consist of isomorphisms $\phi$ between substructures $\mathcal{F}$ of $\mathcal{A}$ (with universe $\left(F_{n, i}(n \in \omega, i<3)\right.$ ) and $\mathcal{G}$ of $\mathcal{B}$ (with universe $\left(G_{n, i}(n \in \omega, i<3)\right)$, which have the following properties:

(A) Each group $F_{n, i}$ is a finite rank pure subgroup of $A_{n, i}$ (and likewise for $\mathcal{G})$.

(B) The groups $F_{n, 0}$ and $F_{n, 1}$ have dual bases, for all $n$ (and likewise for $\mathcal{G})$.

(C) $\quad \sigma_{n}\left[A_{n, 0}\right] \cap F_{n, 2}=\sigma_{n}\left[F_{n, 0}\right]$.

(D) The map $\pi_{n}$ maps $F_{n, 2}$ onto $F_{n+1,0}$, and likewise for $\mathcal{G}$.

We claim that the set $P$ of isomorphisms between substructures with the properties above has the back-and-forth property. Since the cases are symmetric we prove only the case when a new element of $\mathcal{A}$ is chosen.

Suppose that we have an isomorphism $\phi$ between substructures $\mathcal{F}$ and $\mathcal{G}$ with the properties above. We will use the proof we gave for short sequences, referring to the cases covered there as Case S-I, S-II and S-III. The corresponding cases here are:

Case I: We choose $a \in A_{n, 0}-F_{n, 0}$. If $n=0$ then we may proceed as in Case S-I, adding new elements in positions $(0,0),(0,1)$ and $(0,2)$, and extending $\phi$ as we do there. There is no need to add elements at lower levels (that is, at levels $k$ where $k>n$ ).

If $n \geq 1$ we proceed as in Case S-I, extending the groups on level $n$ as we do there (and keeping the same notation). There is no need to add elements on lower levels (by axiom (vii)) but we must extend the groups in position $(n-1,2)$ : Let $k_{1} \in A_{n-1,2}$ be a representative for $a_{2}$, and $l_{1} \in B_{n-1,2}$ be a representative for $b_{2}$. The choice of $a_{2}$ and $b_{2}$ and hypothesis (D) ensure that $k_{1}$ and $l_{1}$ are chosen as in Case S-III(b) (on level $n-1$ ). So we can continue to follow S-III(b) on level $n-1$ to incorporate $k_{1}$ and $l_{1}$. Note that the choice of $a_{2}$ and $b_{2}$ also implies that we need not extend the groups in position $(n-1,0)$. Thus we are done, and $\phi$ may be extended in the obvious manner to an isomorphism with $a$ in its domain. We can check as we did in Section 2 that hypotheses (A) to (D) hold for the extended structures.

Cas e II: We choose $f \in A_{n, 1}-F_{n, 1}$. We follow Case S-II to make the necessary extensions on level $n$, and Case I for level $n-1$ (if $n \geq 1$ ). No extensions are necessary for lower levels.

Cas e III: We choose $k \in A_{n, 2}-F_{n, 2}$. There are two cases:

C a s e III(a): $k \in \sigma_{n}\left[A_{n, 0}\right]+F_{n, 2}$. In this case we may follow the procedure of Case I on level $n$ to add the necessary element of $A_{n, 0}$. The image of $k$ under $\pi_{n}$ is in $F_{n+1,0}$ by axiom (vii), so there is no need to extend the groups at lower levels. 
Case III(b): $k \notin \sigma_{n}\left[A_{n, 0}\right]+F_{n, 2}$. Let $a=\pi_{n}(k)$, an element of $A_{n+1,0}-$ $F_{n+1,0}$. Now follow Case S-I on level $n+1$, extending the groups as we do there. This process produces elements $a_{2} \in A_{n+1,0}$ and $b_{2} \in B_{n+1,0}$; pick $k_{1} \in A_{n, 2}$ and $l_{1} \in B_{n, 2}$ such that $\pi_{n}\left(k_{1}\right)=a_{2}$ and $\pi_{n}\left(l_{1}\right)=b_{2}$. The properties of $a_{2}$ and $b_{2}$ ensure that $k_{1}$ and $l_{1}$ have the properties required by Case S-III(b); so we may continue with the methods of that case on level $n$, to incorporate $k_{1}$ and $l_{1}$. Finally, we may need to add elements to $F_{n, 0}$ to ensure that $k$ itself is incorporated; to do this we may use Case I.

This establishes the "necessity" case of Theorem 3.2. For sufficiency, we first notice that by the same sort of forcing argument as above, two $L_{\infty, \omega^{-}}$ equivalent sequences $\mathfrak{A}$ and $\mathfrak{B}$ have $L_{\infty, \omega}$-equivalent derived sequences. It is then clear from the definitions that $A_{0}$ and $B_{0}$ must have the same length rank.

4. Countable models. We show that countable models of the theory of long sequences exist, and discuss the connection with the "models" of [5]. We finish by showing that the first order theory of long sequences is undecidable.

LEMma 4.1. For any length rank, there is a countable model of the $L_{\infty, \omega}$-theory of long sequences of the given rank.

Proof. Fix a length rank, and let $\mathfrak{A}$ be a standard model of that rank. (The existence of this is established in [4], Theorem XIV.4.9.) We may form its derived sequence $\mathcal{A}$. Now it follows directly from the form of the listed axioms (see the proof of Theorem 3.2) that we may use a Löwenheim-Skolem type argument to find a countable $L_{\infty, \omega}$ submodel $\mathcal{S}$ of $\mathcal{A}$, of the same length rank, and thus by (the proof of) Theorem $3.2, L_{\infty, \omega}$-equivalent to $\mathcal{A}$. Now we may extend the set-theoretic universe to a new universe in which the cardinality of $\mathcal{A}$ is countable. It follows that $\mathcal{S}$ and $\mathcal{A}$ are isomorphic in this new universe. In the ground universe we may carry out the reconstruction procedure (see the proof of Lemma 3.4) on both $\mathcal{A}$ and $\mathcal{S}$ to obtain $\mathfrak{A}$ (by Lemma 3.4) and some structure $\mathfrak{S}$, and since the reconstruction procedure is absolute, we know that in the extended universe $\mathfrak{A}$ and $\mathfrak{S}$ are isomorphic. It follows that they are $L_{\infty, \omega}$-equivalent in the ground model. So $\mathfrak{S}$ is a countable model for the theory of sequences of dual groups (of the given length rank).

In [5] the authors employed structures similar to our long sequences; these are $\omega+1$-sorted structures

$$
\mathfrak{F}=\left(F_{n}(n \in \omega) ;\langle-,-\rangle ; \sigma_{n}(n \in \omega) ; \varrho_{n}(n \geq 2) ; \mathbb{Z}\right)
$$

for the language $\left(\langle-,-\rangle, \boldsymbol{\sigma}_{n}(n \in \omega), \varrho_{n}(n \geq 2)\right)$. The reader should refer to the definitions and results given there; we will comment briefly on the connection with our notion of long sequence. 
In a standard sequence of this type, $F_{0}$ is a dual group, each group $F_{n+1}$ is the dual of $F_{n}$, the bracket and $\sigma$ maps are interpreted as usual, and the map $\varrho_{n}$ is interpreted as restriction, for $n \geq 3$. That is,

$$
\left\langle a, \varrho_{n}(f)\right\rangle=\left\langle\sigma_{n-3}(a), f\right\rangle
$$

for all $a \in F_{n-3}$ and $f \in F_{n}$. The map $\varrho_{2}$ is chosen to be the restriction map arising from the existence of a predual $B$ for $A_{0}$. The existence of the $\varrho_{2}$ maps changes the situation considerably from what we have considered here: for the reasons discussed in Example 1, Theorem 3.2 does not hold for these structures, and of course, having a specified predual $B$ for a given dual group $A_{0}$ misses the point of the program we discussed in the introduction.

However, if one removes the $\varrho_{2}$ map from the language, then the [5] models are really the same as those considered here, since the $\varrho_{n}$ maps for $n \geq 3$ are definable in the reduct of a model to the language of Definition 1.2. One could hope that the axioms for sequences given in [5] axiomatize the $L_{\infty, \omega}$-theory of long sequences, but the following example shows otherwise:

EXAMPLE 2. Let $C$ be a group which is torsionfree but not $\aleph_{1}$-free, and $F$ a separable group so that $F^{* *} / \sigma[F] \cong C$ ([8]). Let $\mathfrak{F}$ be the sequence (of the [5] type without $\varrho_{2}$ ) generated by $F$. Then $\mathfrak{F}$ satisfies the axioms for sequences listed in [5], but in any model $\mathfrak{G}$ of the theory of long sequences, one must have $G_{2} / \sigma_{0}\left[G_{0}\right] \aleph_{1}$-free. So $\mathfrak{F}$ does not model the theory of long sequences.

One can in fact extend the axioms given in [5] to obtain an axiomatization of the theory of long sequences, but since this is somewhat clumsy, and not particularly useful, we do not discuss this here.

We finish by establishing the following theorem.

THEOREM 4.2. The first order theory of any nontrivial sequence of dual groups is undecidable.

Proof. To prove the theorem we embed the first order theory of the ring $\mathbb{Z}$ into the theory of a (long or short) sequence $\mathfrak{A}$. We will assume that $A_{0}$ has rank at least 1 . Fix the element 1 in $\mathbb{Z}, a \in A_{0}$ and $f \in A_{1}$ so that $\langle a, f\rangle=1$. Notice that we can say that " $g$ is a multiple of $f$ " and " $b$ is a multiple of $a$ " by saying that $\operatorname{ker} g=\operatorname{ker} f$ and $\operatorname{ker} a=\operatorname{ker} b$. Given this observation it is easy to define addition and multiplication. First to define addition. Given $n$ and $m$ choose $g, h$ in $A_{1}$ so that $\langle a, g\rangle=n$ and $\langle a, h\rangle=m$; then $n+m=\langle a, g+h\rangle$. To define multiplication is a little trickier. Given $n$ and $m$ choose $b$ a multiple of $a$ so that $\langle b, f\rangle=n$ and choose $g$ a multiple of $f$ so that $\langle a, g\rangle=m$. Then $\langle b, g\rangle=n m$.

The proof may seem circular in that in the multiplicative case we choose $b$ so that $b=n a$. The point is that there is a definable function $\varphi$ (in the 
parameters $a, f)$ so that for $n \in \mathbb{Z}, \varphi(n)=n a$; namely, $\varphi(n)$ is the element $b$ such that $\operatorname{ker} b=\operatorname{ker} a$ and $\langle f, b\rangle=n$.

\section{References}

[1] J. Barwise, Back and forth through infinitary logic, in: Studies in Model Theory, MAA Stud. Math. 8, Math. Assoc. Amer., 1973, 5-34.

[2] S. Chase, Function topologies on abelian groups, Illinois J. Math. 7 (1963), 593-608.

[3] K. Ed a and H. Ohta, On abelian groups of integer-valued continuous functions, their $\mathbb{Z}$-duals and $\mathbb{Z}$-reflexivity, in: Abelian Group Theory, Proc. Third Conf. Oberwolfach, Gordon \& Breach, 1987, 241-257.

[4] P. Eklof and A. Mekler, Almost Free Modules, North-Holland, 1990.

[5] P. Eklof, A. Mekler and S. Shelah, On strongly-non-reflexive groups, Israel J. Math. 59 (1987), 283-298.

[6] E. Ellentuck, Categoricity regained, J. Symbolic Logic 41 (1976), 639-643.

[7] G. Reid, Almost Free Abelian Groups, lecture notes, Tulane University, unpublished, 1968.

[8] G. Schlitt, Sheaves of abelian groups and the quotients $A^{* *} / A$, J. Algebra 158 (1993), 50-60.

DEPARTMENT OF MATHEMATICS

UNIVERSITY COLLEGE OF THE FRASER VALLEY

33844 KING RD.

ABBOTSFORD, BRITISH COLUMBIA V2S 4N2

CANADA

Received 20 August 1992;

in revised form 24 June 1993 